\title{
Evaluation of cell-surface displayed synthetic consensus dengue EDIII cells as a potent oral vaccine candidate
}

\author{
Jyotiranjan Bal ${ }^{1 \dagger}$, Hee-Young Jung ${ }^{1 \dagger}$, Luong Ngoc Nguyen², Jisang Park , Yong-Suk Jang ${ }^{1}$ and Dae-Hyuk Kim ${ }^{\text {* }}$
}

\begin{abstract}
Background: Dengue is a rapidly spreading mosquito borne tropical viral disease affecting hundreds of millions of people across the globe annually. The dengue virus (DENV) includes four genetically distinct serotypes that cause serious life-threatening infections, including dengue hemorrhagic fever/dengue shock syndrome. Dengue vaccine development is complicated by the possibility of vaccine-enhanced severe dengue disease due to antibody-dependent enhancement by pre-existing cross-reactivity, as well as homotypic antibodies. Thus, the development of an efficacious dengue vaccine conferring simultaneous and durable immunity to each of the four DENV serotypes has not yet been developed despite years of research. For mass immunization in deeply affected resource-limited countries, oral vaccination is considered more beneficial than conventional approaches. Therefore, in a continuing effort towards designing economical and potent vaccine candidates, the current study applied yeast surface display technology to develop an oral dengue vaccine candidate using whole recombinant yeast cells displaying the recombinant fusion protein of M cell targeting ligand Co1 fused to the synthetic consensus dengue envelope domain III (scEDIII). Female $\mathrm{Balb} / \mathrm{c}$ mice were orally fed with recombinant yeast cells and immunogenicity in terms of systemic and mucosal immune responses was monitored.
\end{abstract}

Results: Immunofluorescence microscopy with dengue specific antibody and fluorescein isothiocyanate-conjugated anti-mouse IgG antibody clearly showed that recombinant protein Co1-scEDIII-AGA was localized on the cell surface of the respective clones in comparison with scEDIII-Co1 and Mock cells with no fluorescence. Oral dosage applications of surface displayed Co1-sCEDIII-AGA stimulated a systemic humoral immune response in the form of denguespecific serum lgG, as well as a mucosal immune response in the form of secretory immunoglobulin A (slgA). Antigenspecific $B$ cell responses in isolated lymphoid cells from the spleen and Peyer's patches further supported an elevated mucosal immune response. In addition, surface displayed Co1-scEDIII-AGA feeding elicited strong immune responses in comparison with scEDIII-Co1 and Mock following intraperitoneal booster with purified scEDIII antigen.

Conclusions: Surface displayed preparations of Co1-scEDIII-AGA induced strong immunogenicity compared with non-displayed scEDIII-Co1. Prior studies have supported the neutralization potential of scEDIII constructs against all four serotypes. Thus, the oral administration of genetically engineered yeast whole cells displaying biologically active Co1-scEDIII fusion protein without any further processing shows prospective as a potent oral vaccine candidate against dengue viral infection.

Keywords: Dengue, Oral vaccine, scEDIII, Saccharomyces cerevisiae, Surface display, Mucosal immunity

\footnotetext{
*Correspondence: dhkim@jbnu.ac.kr

${ }^{\dagger}$ Jyotiranjan Bal and Hee-Young Jung contributed equally to this work

${ }^{1}$ Institute for Molecular Biology and Genetics, Department of Molecular Biology, Department of Bioactive Material Sciences, Chonbuk National

University, Jeonju, Jeollabuk-do 54896, Republic of Korea

Full list of author information is available at the end of the article
} 


\section{Background}

Dengue, a mosquito-borne viral disease transmitted by female mosquitoes, in particular Aedes aegypti but also $A$. albopictus [1], affects an estimated 50-400 million people annually $[2,3]$. Although considered to be a tropically neglected disease because of its major occurrence in the tropics, approximately $40 \%$ of the world's population is at risk of its transmission. Apart from the increasing number of dengue-affected cases, explosive outbreaks also occur in newer regions, making it a major public health concern. Various factors, including the epidemiology of the four DENV serotypes and the complexity of immunoprotective and immunopathogenic responses following natural infection or vaccination, have created obstacles in the path of dengue vaccine development, despite 70 years of research [4]. Although the first licensed dengue vaccine CYD-TDV [5] has recently been approved, it was associated with an elevated risk of hospitalization for dengue among children younger than 9 years of age [6], and exhibited limited overall vaccine efficacy of 54\% and reduced efficacy of $34 \%$ against DENV2, which is known to cause severe dengue infection and dengue outbreaks [7]. Thus, further studies on the development of potent and effective vaccines are required.

Dengue viruses (DENVs), the etiological agents of dengue disease, are positive-sense RNA viruses belonging to the family Flaviviridae. Comprised of four closely related, but antigenically distinct, serotypes (DENV-1, $-2,-3$, and -4), DENVs have major implications in severe disease consequences, vaccine-induced protection, and epidemic immensity [8]. Dengue infections are categorized as causing asymptomatic fever, dengue fever, and dengue hemorrhagic fever. During secondary infection, antibody-dependent enhancement (ADE) is responsible for the severe manifestations of dengue disease [9]. Therefore, an affordable vaccine with balanced and lasting immunity against all four DENV serotypes is required to prevent dengue infections.

The DENV open reading frame encodes a polyprotein that, upon post-translational processing, results in three structural proteins (capsid [C], membrane [M], and envelope $[E]$ ) and seven non-structural (NS) proteins (NS1, NS2A, NS2B, NS3, NS4A, NS4B, and NS5) [10], among which, the DENV E protein possesses immunomodulatory potential and assists in DENV entry, attachment, and serotype-specific antibody responses in the infected host $[11,12]$. The EDIII domain of the DENV E protein, an immunoglobulin (Ig)-like C terminal domain [13, 14], consists of multiple potent and type-specific neutralizing epitopes [15] and functions as an effective antigen to elicit neutralizing antibodies in experimental animal models and thus represents a novel target for recombinant vaccine development [16]. To address the genetically distinct dengue serotypes, a consensus EDIII (cEDIII) immunogen was created through alignment of sequences from different isolates of the four serotypes of dengue virus, which elicits cross-neutralizing antibodies to block infections by each of the DENV serotypes simultaneously [17]. Yeast expressing scEDIII induced balanced immune responses against all four serotypes upon subcutaneous immunization in mice using purified protein emulsified in complete Freund's adjuvant [18]. Escherichia coli heatlabile enterotoxin (LTB)-conjugated scEDIII produced neutralizing antibodies that elicited both humoral and cell-mediated immune responses [19].

Conventional vaccines are less economical and unsafe, and are therefore unsuitable for mass immunization in resource-limited countries. Oral vaccines have proven to be the best alternative as they avoid the risks commonly associated with conventional vaccines, and confer enhanced mucosal immune responses [20] and systemic responses, as well as being suitable for mass immunizations at a relatively low cost. In general, oral vaccines cause less stress and associated immune suppression for the recipient. Therefore, oral delivery is considered an ideal and easy route to introduce foreign antigens.

Mucosal surfaces play an important role in providing the first line of defense against pathogens. Antigen targeting to the mucosal tissue is essential for effective oral tolerance and initiation of active immune responses. Vaccines delivered into the mucosal immune system can induce effective systemic immune responses simultaneously with mucosal immunity in a manner that is comparable to conventional vaccination [21]. Diverse molecules employed to target vaccine antigens to mucosal and systemic compartments have been characterized. In particular, $M$ cells, the specialized epithelial cells of mucosa-associated lymphoid tissue, are responsible for antigen uptake and their rapid and effective transcytotic activity makes them an attractive target for mucosal vaccine delivery [22]. M cell-specific targeting of the tetravalent dengue antigen (Tet-EDIII) via the Co1 ligand has been performed previously [23]. Co1-conjugated EDIII antigens are also known to be efficiently delivered into Peyer's patches (PP), which facilitates the generation of mucosal immune responses [24]. Furthermore, antibodies induced by the ligand-conjugated EDIII antigen showed effective virus-neutralizing activity.

Saccharomyces cerevisiae, apart from being considered safe (GRAS), is a simple, low cost, and robust eukaryotic expression system with known tools of genetic manipulation that possesses inherent advantages of eukaryotic post-translational modifications and secretion, and the cells are suitable to be taken up by antigen-presenting cells (APCs) [25]. Furthermore, from the vaccine point of view, recombinant $S$. cerevisiae are potent transporters 
of target protein and expression vector DNA into dendritic cells, triggering antigen-specific CD4+ and CD8+ immune responses in vivo [26, 27]. Oral immunization of recombinant $S$. cerevisiae activates potent innate, as well as adaptive, $\mathrm{T}$ cell immune responses to the target antigen $[19,28]$. The above properties make S. cerevisiae a potent nonpathogenic vaccine-delivery vehicle.

Yeast surface display technology represents an efficient approach for antigen presentation in the cell. It is commonly used for functionalizing yeast cells for a variety of biotechnological applications, including generation of whole-cell biocatalysts, antimicrobial agents, and oral vaccines [29]. Integration of the protein of interest to the $\mathrm{C}$ - or N-terminus of an anchor protein on the cell surface of $S$. cerevisiae typically results in the display of up to 100,000 copies of the fusion protein [30]. Several studies have proposed the suitability of antigen-displaying yeast for preventative or therapeutic oral vaccines [31, 32]. Pichia pastoris surface displayed hemagglutinin protein from a highly pathogenic avian influenza virus, subtype $\mathrm{H} 5 \mathrm{~N} 1$, and produced virus neutralizing antibodies upon oral administration [33]. A neutralizing epitope of ApxIIA exotoxin of Korean Actinobacillus pleuropneumoniae displayed on the $S$. cerevisiae cell surface elicited strong immune responses when administered orally and was sufficient to protect against pathogen infection [34]. Oral delivery of $S$. cerevisiae cells displaying Enolp on their cell surfaces protected $60 \%$ of mice against candidiasis [35]. The yeast surface display system is also advantageous as it provides antigenic proteins more rapidly and conveniently than conventional approaches of vaccine production.

Taking into consideration the immunogenic efficacy of scEDIII, the efficient mucosal targeting, elicitation of immune responses by Co1, and advantages of the surface display technology of $S$. cerevisiae, we evaluated the immunogenicity of the fused recombinant antigen surface displayed preparations of $S$. cerevisiae as an oral dengue vaccine candidate in a female BALB/c mouse model.

\section{Methods}

\section{Animal housing and ethics statement}

Female BALB/c mice, aged 5 weeks, were procured from the Charles River Laboratories through Orient Bio, Inc. (Sungnam, Korea) and maintained under general specific pathogen-free conditions. Six mice per filtertop microisolator cage were housed in a temperature- and humiditycontrolled room. Mice were acclimated for approximately 1 week prior to initiation of the oral feeding experiment. Experimental procedures involving laboratory animals strictly adhered to the guidelines established by the Institutional Animal Care and Use Committee of the
Chonbuk National University (Approval Number: CBU 2015-0004).

\section{Reagents}

An anti-dengue virus primary monoclonal antibody $(\mathrm{mAb})$ was procured from LifeSpan BioSciences, Inc. (Seattle, WA, USA). This antibody recognizes all four dengue virus serotypes (DENV-1, DENV-2, DENV-3 and DENV-4) of the genus Flavivirus. An anti-mouse IgG (whole molecule)-fluorescein isothiocyanate (FITC) antibody produced in goat was purchased from SigmaAldrich (St. Louis, MO, USA).

\section{Construction of fusion protein expression vectors}

To construct the expression vector for surface display of the recombinant protein scEDIII, the amylase 1A (Ramy1A) signal peptide, the M-cell specific ligand (Co1) gene, target gene fragment scEDIII, and an anchor DNA fragment containing the $3^{\prime}$ half of the $\alpha$-agglutinin gene (AGA1-C320) encoding the C-terminal 320 amino acids [36] were fused sequentially by overlap extension polymerase chain reaction (PCR) using primers listed in Table 1 and DNA polymerase from Takara Bio Inc. (Otsu, Japan). S. cerevisiae codon-optimized scEDIII gene [18] was amplified from pUC19 harboring scEDIII. Furthermore, for the construction of the control strain lacking surface display of the scEDIII antigen, the Col gene and scEDIII alone were fused through overlap extension PCR using the primers listed in Table 1. In addition, BamHI and SalI restriction sites were included at the $5^{\prime}$ and $3^{\prime}$ ends of both the fusion constructs, respectively, to facilitate subsequent cloning. The fusion constructs were cloned into the yeast episomal shuttle vector, pYEGPDTER, to construct pYEG-R-Co1-scEDIII-AGA-TER with glyceraldehyde-3-phosphate dehydrogenase (GPD) as the promoter and galactose-1-phosphate uridyl transferase (GAL7) as the terminator (Fig. 1). The resulting clones were confirmed through restriction enzyme digestion and DNA sequencing. The expression host $S$. cerevisiae

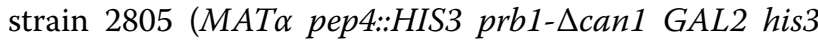
ura3-52) [37] was transformed by pYEG-R-Co1-scEDIIIAGA-TER and pYEG-scEDIII-Co1-TER using the lithium acetate method [38]. Empty vector pYEGPD-TER-transformed yeast (termed Mock) was used as a negative control.

\section{Northern blot analysis to confirm expression of the target gene and selection of efficient clones}

As described previously [18], total yeast RNA was extracted from the selected clones, separated on a $1.2 \%$ formaldehyde-agarose gel, transferred to an Amersham Hybond $^{\mathrm{TM}}$ membrane, and hybridized with an $\alpha-\left[{ }^{32} \mathrm{P}\right]-$ labeled probe generated using a random labeling kit 
Table 1 Oligonucleotide primers used in this study

\begin{tabular}{|c|c|}
\hline Primers & Sequence $\left(5^{\prime}-3^{\prime}\right)^{a}$ \\
\hline \multicolumn{2}{|c|}{ Co1-scEDIII-AGA construction } \\
\hline Ramyl-F & 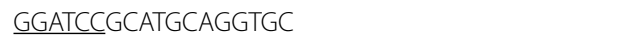 \\
\hline Ramyl-Co1 OR & TAGCTGGTAGTTGATGGAAAGACCCGGCTGTCAAGT \\
\hline Co1-scEDIII F & TCAACTACCAGCTAGAAGTCCACTACCAAAAGGAATGTCT \\
\hline scEDIII-AGA OF & TAAAAAGGGTTCCTCAGCCAAAAGCTCT \\
\hline scEDIII-AGA OR & AGAGCTTTTGGCTGAGGAACCCTTTTTA \\
\hline AGA-R & GTCGACGCTTAGAATAGCAGGTACGACAA \\
\hline \multicolumn{2}{|c|}{ scEDIII-Co1 construction } \\
\hline scEDIII-Co1-F & CGGGATCCCGATGAAAGGAATGTCTTACGCA \\
\hline scEDIII-Co1-R & $\begin{array}{l}\text { GTCGACCTATGGTAGTGGACTTCTAGCTGGTAG TTGATG } \\
\text { GAAAGATGAGGAACCCT }\end{array}$ \\
\hline
\end{tabular}

a Underlined nucleotides indicate engineered restriction sites used in cloning

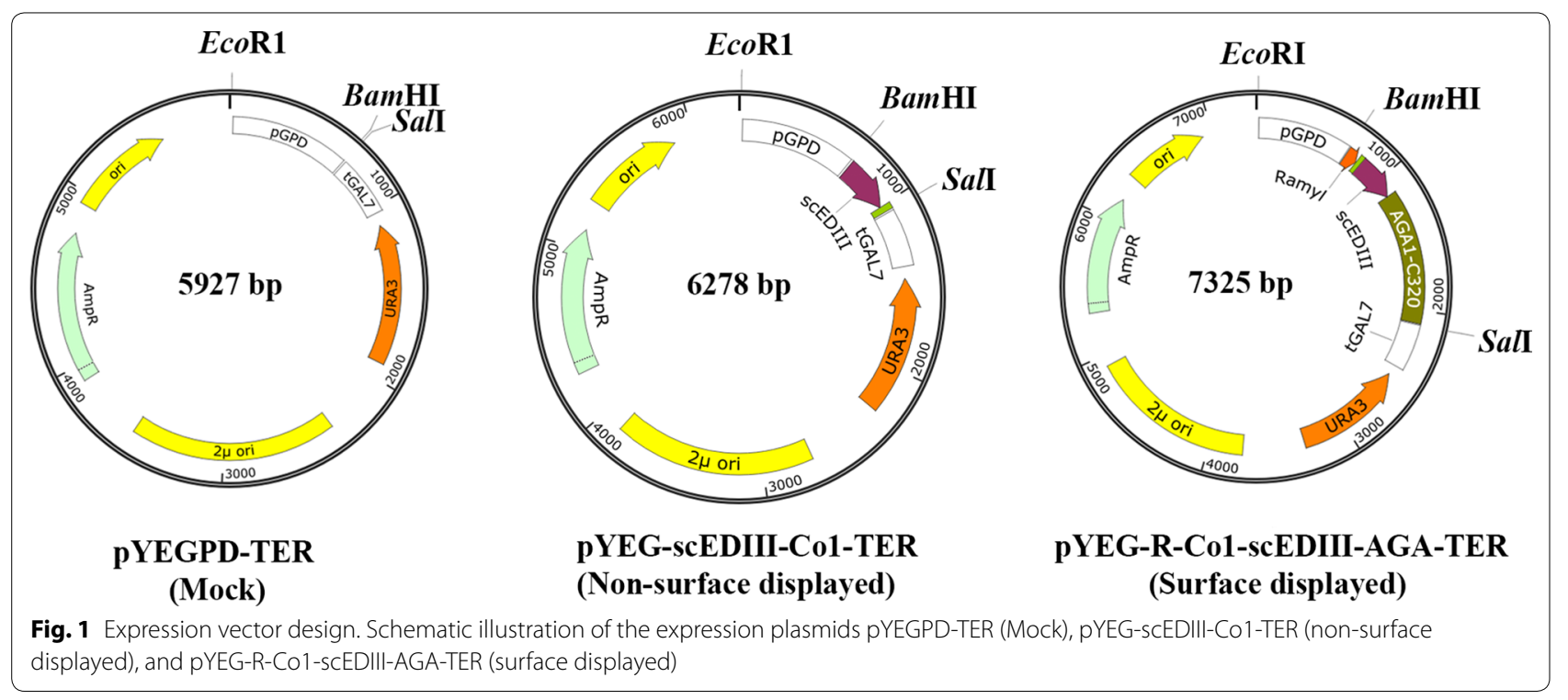

(Amersham Pharmacia Biotech, Piscataway, NJ, USA). The developed blot visualized the expression pattern of scEDIII-Co1 and Co1-scEDIII-AGA along with S. cerevisiae GPD as a reference.

\section{Immunostaining and fluorescence microscopy}

Immunofluorescence labeling of recombinant yeast cells was performed to confirm the expression and surface display of Co1-scEDIII-AGA through a slightly modified method, as described previously [36, 39]. Cultivated recombinant yeast cells $(48 \mathrm{~h})$ were washed three times with phosphate-buffered saline (PBS). The pellet was resuspended in PBS containing 1\% bovine serum albumin (BSA) and incubated for $30 \mathrm{~min}$ at room temperature. Cells were then washed three times with PBS, resuspended in PBS containing 3\% BSA and polyclonal anti-dengue mouse IgG (1:100), and incubated at room temperature for $2 \mathrm{~h}$. Next, cells were washed as described above, the pellet was resuspended in PBS containing 3\% BSA and secondary antibody, FITC-conjugated goat anti-mouse IgG (1:50; Sigma-Aldrich), and incubated at room temperature for $1 \mathrm{~h}$. After washing, the cells were observed using confocal laser scanning microscopy (Carl Zeiss, Zena, Germany).

Culture conditions and target protein expression analysis The $S$. cerevisiae transformants containing pYEG-R-Co1scEDIII-AGA-TER and pYEG- scEDIII-Co1-TER were cultured and maintained in uracil-deficient (ura ${ }^{-}$) selective medium, as described previously [19]. The E. coli strains were maintained in Luria-Bertani broth with the appropriate antibiotics. 
For target protein expression in non-surface displayed yeasts, cell-free extracts (CFEs) were prepared, as described previously [18]. The cell wall fraction (CWF) was extracted and analyzed to confirm expression of the target protein at the cell surface. Cultured cells were resuspended in $200 \mu \mathrm{L}$ of extraction buffer (50 mM Tris-Cl, pH 8.0; 2\% SDS; 10 mM DTT; $0.1 \mathrm{M}$ EDTA) and boiled for $30 \mathrm{~min}$, followed by centrifugation at top speed for $20 \mathrm{~min}$ at $4{ }^{\circ} \mathrm{C}$. After suspension, samples were boiled for $30 \mathrm{~min}$ and centrifuged at top speed for $20 \mathrm{~min}$ at $4{ }^{\circ} \mathrm{C}$. After centrifuging, the supernatant was transferred into a $1.5 \mathrm{~mL}$ EP tube. Protein concentration was determined using the Bio-Rad Protein Assay Kit (Bio-Rad, Hercules, CA, USA). Sample aliquots of the CFEs and CWF were separated by SDS-polyacrylamide gel electrophoresis and transferred onto Hybond-C Extra nitrocellulose filter membranes (Hybond, Amersham Pharmacia Biotech). Primary anti-dengue virus $\mathrm{mAb}$ (LifeSpan BioSciences, Inc.) that recognized scEDIII was used to detect target proteins. After incubation with goat anti-mouse IgG alkaline phosphatase conjugate (Sigma-Aldrich), the blots were developed by BCIP/NBT in TMN buffer (100 mM Tris, $\mathrm{pH} 9.5 ; 5 \mathrm{mM} \mathrm{MgCl}_{2}$; and $100 \mathrm{mM} \mathrm{NaCl}$ ). Purified protein extract from the BL21 $E$. coli strain expressing scEDIII [16] was used as a positive control.

\section{Preparation of oral doses for vaccination}

In accordance with the successful elicitation of an immune response due to the oral dose of $1.6 \mathrm{~g}$ fresh weight of recombinant yeast cells in our previous study [19], the same dose was used for the oral vaccination of mice. Briefly, $\sim 20 \mathrm{~g}$ fresh weight of recombinant yeast cells was harvested from $640 \mathrm{~mL}$ of culture and subsequently divided into aliquots containing $1.6 \mathrm{~g}$ of recombinant yeast cells for a single dose.

\section{Oral immunization and blood and fecal sampling}

The oral immunization strategy and schedule were followed as described previously with minor modifications [19]. Briefly, mice groups were fasted overnight (water was provided ad libitum). Oral feeding with whole recombinant yeast cell preparations was performed using a 1-mL syringe fitted with an oral feeding needle. The three groups consisting of six BALB/c mice were fed separately with Mock, Co1-scEDIII-AGA, or scEDIII-Co1. Each BALB/c mouse was administered a total of six doses, with each dose consisting of $1.6 \mathrm{~g}$ fresh weight of cells, resuspended in a final volume of $2.4 \mathrm{~mL}$ of PBS, split equally, and orally gavaged four times every alternate week, as depicted in Fig. 2 with minimum distress. To investigate the memory response, an intraperitoneal injection of $20 \mu \mathrm{g}$ of alum-adsorbed purified $E$. coli-expressed scEDIII was administered to each immunized mouse 3 weeks after the last oral immunization. Blood and fecal pellets were collected periodically from each mouse at day (d) 18, d25, and d32 after the last oral immunization (Fig. 2). Blood was collected through retroorbital bleeding, maintained at room temperature for $1 \mathrm{~h}$ to clot, and kept overnight at $4{ }^{\circ} \mathrm{C}$ to facilitate clot retraction before serum was recovered for storage at $-20^{\circ} \mathrm{C}$. Extracts from the collected fecal matter were prepared in PBS for enzyme-linked immunosorbent assay (ELISA) analysis. To observe memory responses, weeks after the final immunization, intraperitoneal injection of a booster dose of E. coli expressed purified scEDIII antigen was administered. Retro-orbital bleeding was then performed after 3 days of injection.

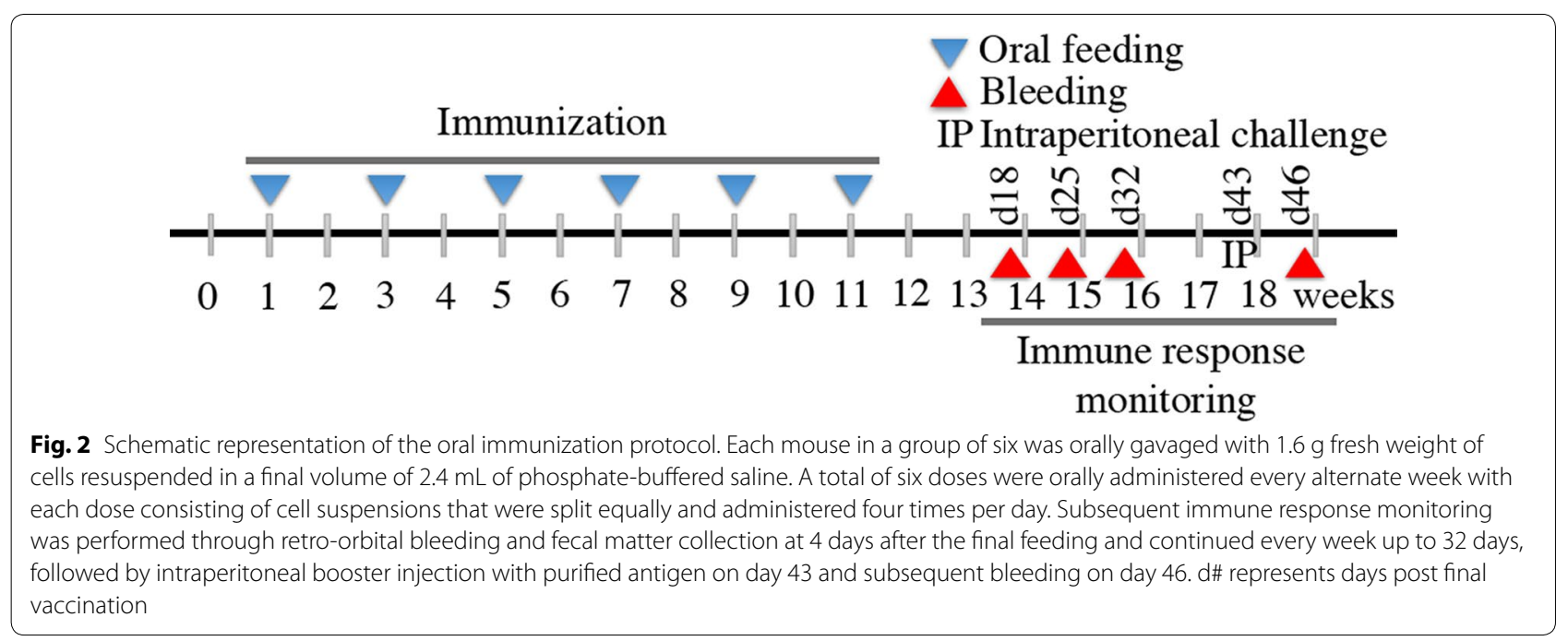




\section{ELISA detection of anti-dengue antibodies}

Changes in humoral immune responses upon oral administration of recombinant yeast cells were analyzed by estimating the antibodies induced through indirect ELISAs using NUNC Maxisorp 96-well ELISA plates coated with $100 \mathrm{ng} /$ well of $E$. coli-expressed recombinant scEDIII protein. The plates were washed three times with PBS $+0.05 \%$ Tween 20 and blocked with $1 \%$ BSA in PBS for $2 \mathrm{~h}$ at $37^{\circ} \mathrm{C}$. Following the washes, twofold serial dilutions were performed after adding $100 \mu \mathrm{L} /$ well of sera from immunized mice (the starting dilution points were 1:25 for serum IgG and 1:2 for fecal sIgA), and the plates were incubated overnight at $4{ }^{\circ} \mathrm{C}$. Alkaline phosphatase (AP)-conjugated secondary antibodies (anti-mouse IgG or IgA; Sigma-Aldrich) diluted 1:5000 in PBS containing $0.5 \%$ BSA were added and incubated for $2 \mathrm{~h}$ at $37^{\circ} \mathrm{C}$ followed by a washing step. To detect the response, $100 \mu \mathrm{L} /$ well of phosphatase substrate (S0942, Sigma-Aldrich) was added and incubated for $15 \mathrm{~min}$ at room temperature. The reaction was stopped with $2 \mathrm{M} \mathrm{H}_{2} \mathrm{SO}_{4}$ and the optical density was measured at $405 \mathrm{~nm}$ using a microplate reader (Multiskan ${ }^{\mathrm{TM}}$ GO Microplate Spectrophotometer, Thermo Fisher Scientific Inc., Waltham, MA, USA).

\section{Enzyme-linked immunosorbent spot (ELISPOT) assay}

ELISPOT assay to enumerate IgG or IgA secreting cells in lymphocytes isolated from the spleen and PPs of immunized mice was conducted as described previously $[40,41]$. The PPs were carefully excised from the small intestines of two mice randomly selected from a group of six on $\mathrm{d} 39$ before booster immunization and further dissociated into single $\mathrm{T}$ cells by stirring with collagenase $\mathrm{D}(0.5 \mathrm{mg} / \mathrm{mL})$ and DNase I $(100 \mu \mathrm{g} / \mathrm{mL})$ for $60 \mathrm{~min}$ at $37^{\circ} \mathrm{C}$.

\section{Statistical analysis}

The statistical significance of the difference between the experimental parameters was determined using the twotailed Student's $t$-test and analysis of variance, wherever indicated, using GraphPad Prism Mac version 6.0e. $p$-values $<0.05$ were considered to indicate significance.

\section{Results}

\section{Creation of an S. cerevisiae strain displaying scEDIII on its cell surface}

To create an S. cerevisiae strain displaying recombinant scEDIII protein on its cell surface, the plasmid pYEGR-Co1-scEDIII-AGA-TER was constructed, as described in "Methods" (Fig. 1). This expression plasmid is a multicopy plasmid for expression of the fusion gene containing the secretion signal sequence Ramy1A under control of the GPD promoter. The protein of interest is secreted due to the Ramy1A signal sequence and is anchored to the cell surface via AGA1. Following transformation of the above plasmid into $S$. cerevisiae strain 2805 , more than 20 transformants were randomly selected on ura- medium and were further examined using colony PCR and E. coli back transformation to confirm the presence of the corresponding recombinant plasmid. To compare and analyze the beneficial effects due to the surface display of the target antigen, an S. cerevisiae strain expressing only the $\mathrm{M}$ cell targeting ligand Co1 conjugated to scEDIII and another expressing empty vector only, also referred to as Mock, respectively, were created as controls.

Northern blot analysis of the selected transformants using fusion gene constructs revealed the accumulation of scEDIII transcripts in all transformants (Fig. 3a, b). Among the transformants harboring Co1-scEDIII-AGA, one was selected (\#7 strain showing the highest transcript level), whereas among transformants harboring scEDIII-Co1, \#8 based on the high expression level relative to the internal control (GPD) was selected for subsequent experiments.

\section{Western blot analysis to confirm the expression of recombinant scEDIII}

Western blot analysis of CFEs of the strain harboring pYEG-Co1-scEDIII-TER showed a corresponding scEDIII-Co1 protein band at $\sim 15 \mathrm{kDa}$ confirming the expression of Co1-scEDIII (Fig. 3c). To further verify the expression of recombinant scEDIII and its association with the yeast cell wall, the selected $S$. cerevisiae transformant strain \#7 harboring the pYEG-R-Co1-scEDIII-AGA-TER plasmid was cultured, followed by isolation of the CWF and subsequent extraction of cell wall proteins (CWPs). Isolation of the CWF from negative controls, such as Mock and wild-type cells, were also extracted. Western blot analysis to confirm the identity of the recombinant protein using anti-dengue antibody revealed that the CWPs of cells harboring pYEG-R-Co1scEDIII-AGA-TER showed a band corresponding to the size of the fusion protein of Co1-scEDIII-AGA with the expected size of $\sim 50 \mathrm{kDa}$ (Fig. 3d), whereas no corresponding band was found in the culture filtrate of the transformant cells or in the cell fractions of Mock cells or wild-type cells, confirming the anchorage of the target protein Co1-scEDIII-AGA to the yeast cell wall.

\section{Fluorescence labeling for detection of the surface displayed recombinant protein Co1-scEDIII-AGA}

To confirm the secretion and targeting to the cell surface of the expressed Co1-scEDIII-AGA, recombinant yeast cells in the exponential growth phase were labeled with FITC-conjugated antibodies followed by visualization using confocal laser scanning microscopy (Fig. 4). The cell walls of recombinant strains harboring pYEG-R-Co1-scEDIII-AGA-TER 


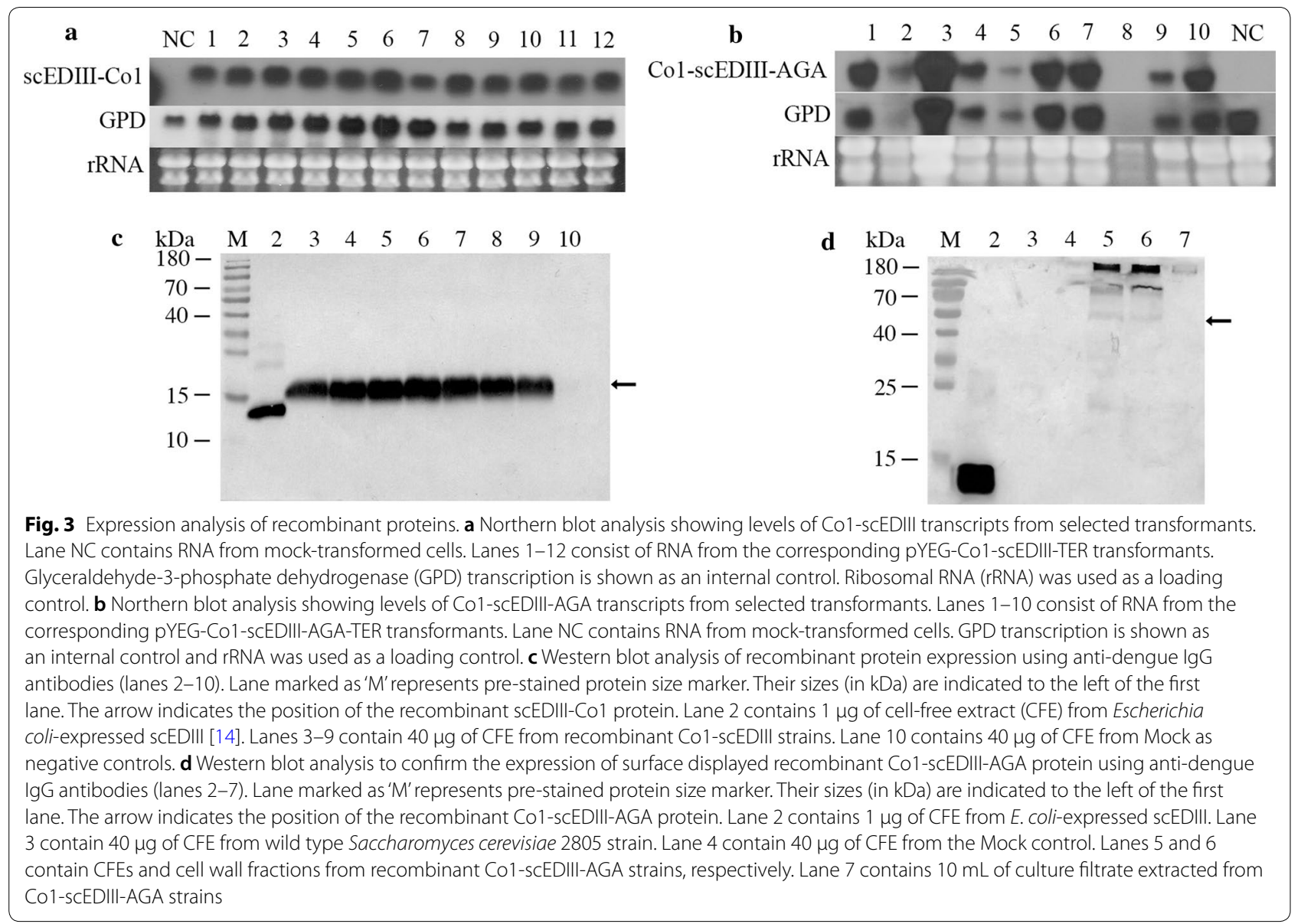

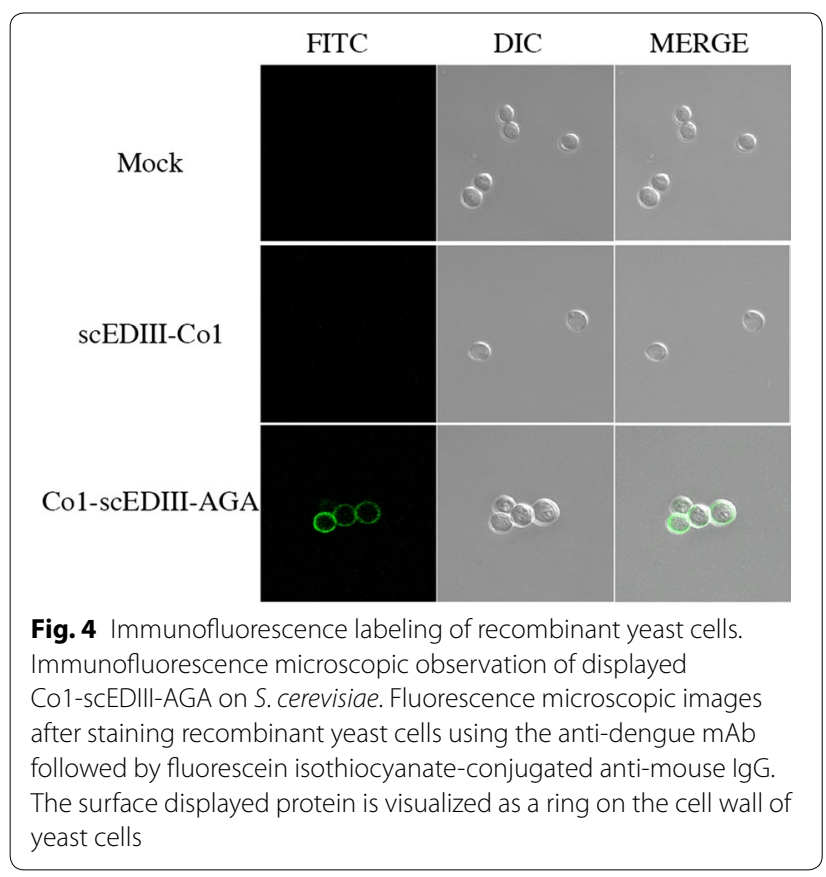

fluoresced and were visualized as a ring, in contrast to no fluorescence in the mock transformant and the strain harboring pYEG-scEDIII-Co1-TER. This confirms the specificity of the immunofluorescence labeling and correct localization of the Co1-scEDIII-AGA antigen on the yeast cell surface. The absence of fluorescence in other parts of the cell indicates no cross-reactivity of dengue antibody with recombinant yeast cells.

\section{Surface displayed Co1-scEDIII elicits a systemic humoral immune response upon oral immunization}

M-cell targeting Co1 ligand is an effective mucosal targeting molecule and S. cerevisiae cells have multiple adjuvant properties apart from being GRAS, thereby making them an ideal combination to elicit immune responses. Thus, we explored the immunogenicity of the surface displayed yeast that expressed Co1-scEDIII-AGA through oral administration. Mice immunized with Co1-scEDIIIAGA showed significantly higher scEDIII-specific IgG antibody titers compared to Mock, which showed no dengue specific IgG antibodies (Fig. 5). Furthermore, 


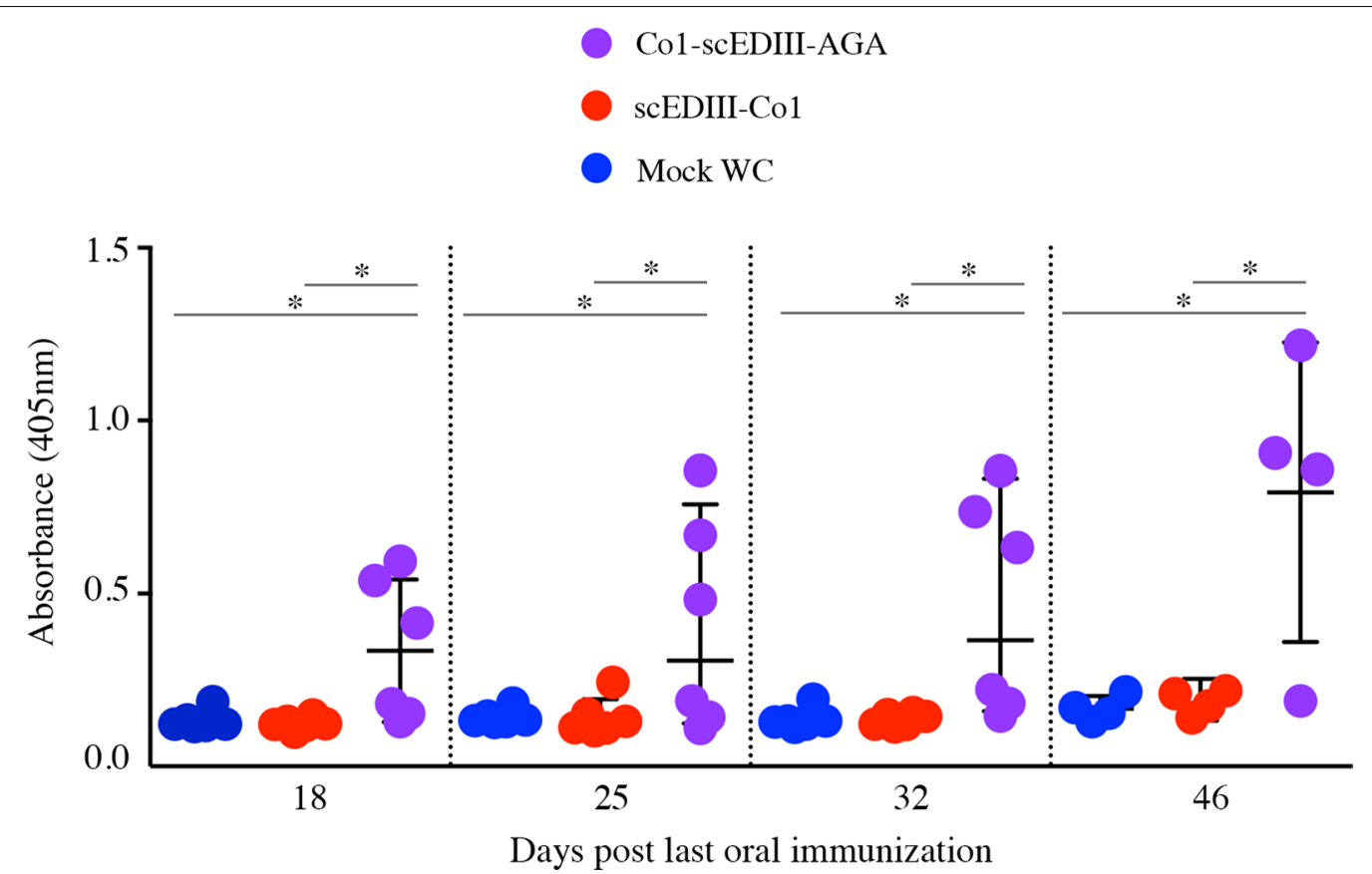

Fig. 5 Humoral lgG responses in orally immunized mice. Dengue-specific serum lgG was induced in mice that received oral administration of scEDIII-Co1 and Co1-scEDIII-AGA, and this induction was observed at 4 days after the last immunization until day 32, as measured at weekly intervals. On day 46 , following booster immunization with purified $E$. coli expressed scEDIII protein, dengue-specific serum IgG levels were also observed. An unpaired Student's t-test was used to calculate $p$-values, and ${ }^{*} p<0.05$ indicates significant differences between the groups

Co1-scEDIII-AGA immunized mice showed significantly higher dengue specific antibody titers compared to non-surface displayed scEDIII-Co1 immunized mice. However, mice immunized with scEDIII-Co1 showed no significant levels of dengue-specific IgG compared with those immunized with Mock. The detected immune responses showed prime-boost-dependent kinetics, with antibody titer sharply rising following the second boost.

\section{Oral immunization of surface displayed Co1-scEDIII elicited} a mucosal immune response

The effectiveness of an oral vaccine depends on activation of the mucosal immune response system. Therefore, the mucosal immune response in orally immunized mice was estimated through dengue specific fecal sIgA antibody titers with ELISA. Mice immunized with surface displayed Co1-scEDIII-AGA elicited scEDIII-specific fecal sIgA. However, the dengue specific sIgA levels for mice immunized with surface displayed Co1-scEDIIIAGA were higher on $\mathrm{d} 25$ post last oral immunization, but were not significantly different from mice immunized with scEDIII-Co1 or mock transformant (Fig. 6). These results suggest that scEDIII was targeted to the mucosal system via surface displayed cells, despite the lower mucosal immune response.

\section{ELISPOT assay enumerates the number of IgG-} and sigA-secreting cells upon oral immunization of surface displayed Co1-scEDIII

Apart from heterogeneity, conventional ELISA revealed antibody titers that were below threshold levels. To confirm further the scEDIII-specific mucosal immune responses, we performed ELISPOT assays, which are much more sensitive than conventional ELISAs, on lymphocytes isolated from mucosal tissues such as the spleen and PPs from immunized mice. Briefly, on d39 before booster immunization, two randomly selected mice were sacrificed from each group, and their spleens and PPs were retrieved. Lymphocytes were isolated from the tissues and probed for scEDIII-specific IgG- and IgA-secreting cells using ELISPOT assays. The number of scEDIII-specific antibody forming cells (AFCs) in the form of spot-forming cells in the spleen and PP lymphocytes differed considerably between surface displayed Co1-scEDIII-AGA administered mice and their scEDIII-Co1 and Mock-administered counterparts (Fig. 7). Furthermore, comparatively higher numbers of scEDIIIspecific IgG AFCs in splenic lymphocytes and scEDIIIspecific IgA AFCs in the PP lymphocytes were observed in the surface displayed Co1-scEDIII-AGA compared to scEDIII-Co1 mice. The mucosal cells from Mock-fed mice showed no scEDIII-specific IgG AFCs or IgA AFCs 
Mock WC

scEDIII-Co1

Co1-scEDIII-AGA

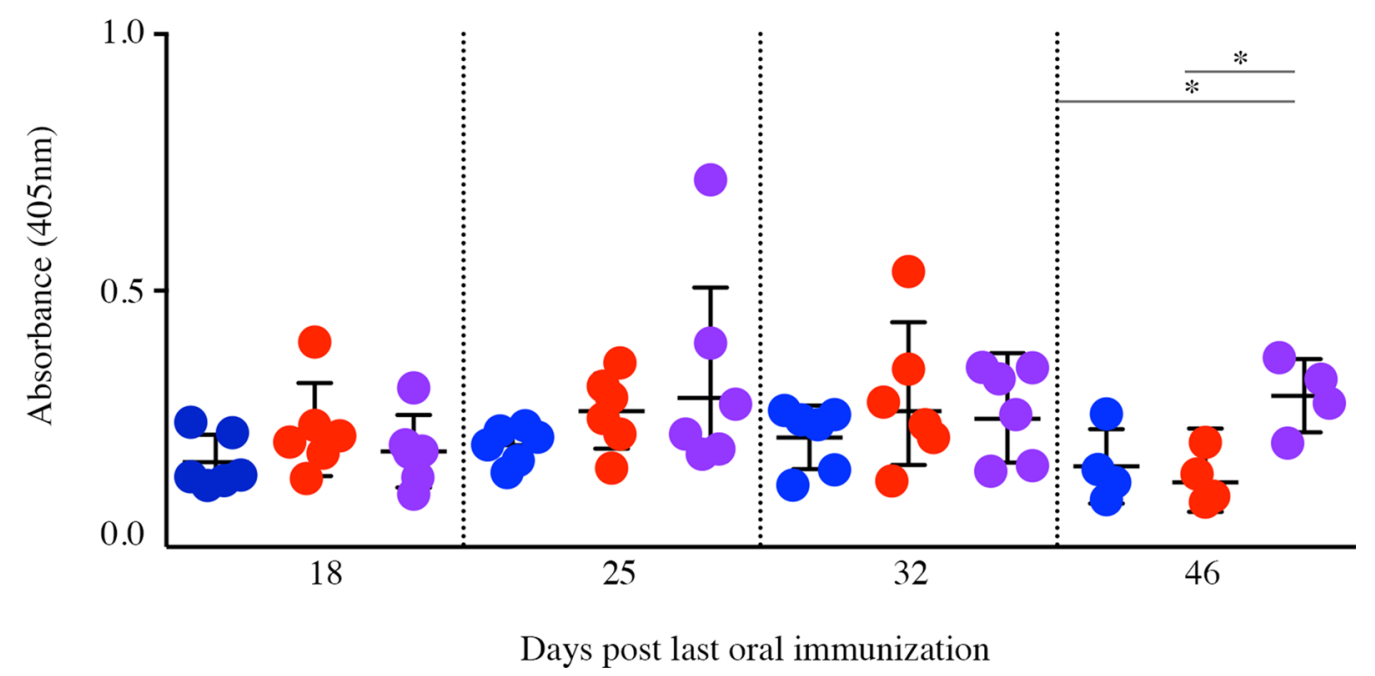

Fig. 6 Mucosal slgA responses upon oral immunization. scEDIIl-specific fecal slgA was induced in mice immunized orally with recombinant scEDIII-Co1 and Co1-scEDIII-AGA at 4 days after the last immunization until day 32, observed at weekly intervals. Dengue-specific fecal slgA levels were also observed on day 46, 3 days after booster immunization with purified E. coli expressed scEDIII protein. An unpaired Student's $t$-test was used to calculate $p$-values, and ${ }^{*} p<0.05$ indicates significant differences between the groups

in mucosal cells. These results suggest that the enhanced levels of scEDIII-specific serum IgG and fecal IgA were caused by efficient targeting to the mucosal immune system due to the display of the target antigen on yeast cell surface and presence of $\mathrm{Co} 1$ on the fusion protein.

\section{Oral immunization elicits an elevated immune response upon booster immunization}

Long-term memory response upon antigenic challenge is an essential attribute of an efficient vaccine. To determine whether a memory response had been established as a result of oral immunization, we evaluated serum IgG levels following administration of an intraperitoneal booster injection of $20 \mu \mathrm{g}$ of alum-adsorbed purified $E$. coli-expressed scEDIII to each immunized mouse on $\mathrm{d} 43$ following the last oral immunization. The secondary immune response due to oral immunization of surface displayed Co1-scEDIII-AGA was significantly higher compared to both Mock and scEDIII-Co1 upon intraperitoneal antigenic booster dose (Figs. 5, 6). Both IgG and sIgA titers with surface displayed Co1-scEDIIIAGA were significantly higher than those of the booster dose immunized Mock and scEDIII-Co1, respectively. These results suggest that a strong memory response may have been elicited upon oral immunization with surface displayed Co1-scEDIII-AGA in contrast to that of nondisplayed scEDIII-Co1.

\section{Discussion}

Dengue is considered a rapidly spreading fatal disease across the globe, and an efficacious and economical vaccine is required for mass immunization to prevent dissemination. In our previous studies on recombinant dengue vaccine development, we first confirmed targeted delivery of an $\mathrm{M}$ cell-specific $\mathrm{Co1}$ ligand-conjugated recombinant tetravalent gene comprising the amino acid sequences of dengue envelope domain III (Tet-EDIII) from four serotypes into $M$ cells in PPs [23]. Furthermore, yeast-expressed scEDIII possessed a balanced immune response against all four serotypes [18]. Subsequently, we evaluated $S$. cerevisiae-expressed $E$. coli LTBconjugated scEDIII as an oral vaccine candidate in the form of whole cells (WCs) or CFEs, both of which elicited mucosal as well as cell-mediated immune responses, with the exception that the immune response due to WCs was lower than that of CFEs [19]. Earlier observations showed that WCs have an additional advantage compared to CFEs in the form of oral vaccines. Soluble antigens do not sufficiently penetrate the mucus layer of the intestine. As a result, these antigens are taken up marginally by APCs and generate tolerance [42]. Immune 

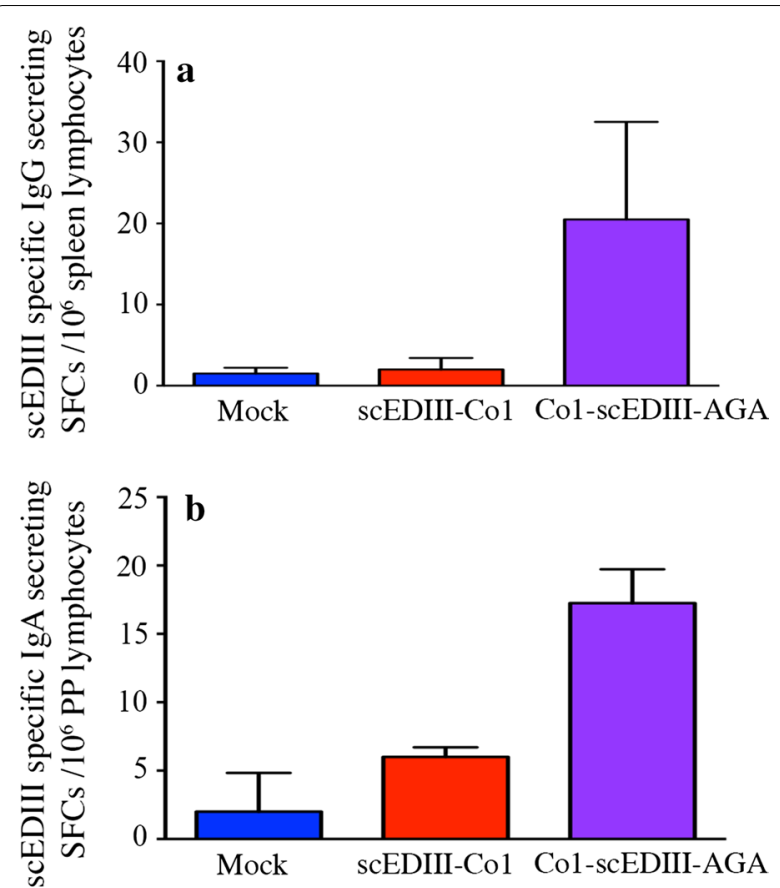

Fig. 7 Frequency of antigen-specific immune cells in orally immunized mice. Enzyme-linked immunosorbent spot assay showing (a) scEDIII-specific lgG and (b) scEDIII-specific IgA antibody spot forming cells in lymphocytes isolated from spleens and Peyer's patches of immunized mice, respectively. Each group represents two mice analyzed individually in triplicate. This is a representative result of two independent experiments showing similar results

responses against the wild-type yeast cell were shown to be higher than that of the hepatitis $B$ surface antigen protein HBS3 [43]. Therefore, considering the advantages of WCs, an attempt was made in the present study to further improve the efficacy of the WC formulation using a combination of two strategies, one of which includes yeast surface display technology, which presents the antigenic proteins more rapidly and conveniently, and the second of which includes the use of mucosal $\mathrm{M}$ cell targeting ligand $\mathrm{Co} 1$ for efficient mucosal targeting.

The EDIII domain of the DENV E protein is a known effective antigen that elicits neutralizing antibodies in experimental animal models and represents a critical target for recombinant vaccine development [16]. Recent studies have shown that LTB-conjugated scEDIII expressed yeast upon oral feeding elicited both humoral and mucosal immune responses in mice [19]. A tetravalent virus-like particle vaccine designed to display the EDIII domain was shown to induce multi-serotype neutralizing antibodies in mice and macaques, which confer protection against ADE [44]. Previous studies have also shown that the consensus EDIII expressed in tobacco plants induced a significant cellular immune response in the form of interferon- $\gamma$ production and polyfunctional $\mathrm{T}$ cells in both the CD4+ and CD8+ compartments [45]. Based on these studies and considering the diversity between serotypes, the scEDIII construct was used in the current study and retained its antigenic characteristics despite being conjugated with the $\mathrm{M}$ cell targeting ligand $\mathrm{Co} 1$ and anchored to the yeast cell surface. Western blot analysis using anti-dengue $\mathrm{mAb}$ confirmed the specificity and predicted molecular weight of the recombinant construct.

For an effective oral vaccine, elicitation of a mucosal immune response is critical. The mucosal $\mathrm{M}$ cells are the major portal that take up luminal antigens and initiate antigen-specific immune responses [46]. These $M$ cells regulate tolerogenicity of the mucosal environment conjugated with efficient presentation of the antigen to the lymphoid organ for processing. Oral vaccines exploit advantages of $M$ cells via $M$ cell-targeting ligands that have the potential to deliver ligand-conjugated antigens into mucosal lymphoid organs and evoke conjugated antigen-specific systemic and mucosal immune responses [47]. The $\mathrm{M}$ cell targeting ligand Co1A conjugated to the $\mathrm{C}$-terminus of EDIII was reported to enhance EDIII-specific immune responses in systemic and mucosal compartments by T-cell stimulation [24]. The current study includes $\mathrm{M}$ cell targeting ligand $\mathrm{Co} 1$ conjugated to the target antigen scEDIII to achieve efficient mucosal targeting. Its precise conjugation and expression was explored through western and northern analyses.

The recombinant construct Co1-scEDIII-AGA has been successfully expressed and anchored to the yeast cell surface, as validated through FITC fluorescent labeling using anti-Dengue mAb. Yeast surface display, the platform currently being used to incorporate recombinant proteins into the cell wall, has achieved significant success with countless applications in biotechnology, including bioconversion, edible vaccines, bioremediation, and bioseparation [43, 48-50]. In addition to the convenience of production, which lacks expensive and tiresome purification processes, yeast cell wall components known to possess natural adjuvant activity act synergistically as a delivery vehicle and an adjuvant enabling the recombinant proteins to be more immunogenic [51]. Furthermore, antigens expressed and displayed on the yeast cell surface possess dual advantages when used as oral vaccines, which includes easy access to antibodies and the target antigen being presented on a large surface in the vicinity of mucosal tissue for improved adsorption $[42,43]$. The ability to resist thermal and chemical denaturation and proteolytic degradation in oral vaccines is essential. Surface display of the target protein increases structural stability, inducing thermal stability of the target protein [52]. Thus, S. cerevisiae expressing 
recombinant antigenic determinants on its surface was considered a good oral vaccine candidate.

Recombinant protein Co1-scEDIII-AGA being successfully displayed on the yeast cell surface and retaining its functional properties led us to further investigate its immunogenicity to characterize it as a vaccine candidate. We found that surface displayed Co1-scEDIII-AGA developed anti-dengue IgG titers at 18 days after the last immunization, showing elicitation of a humoral response that differed significantly from controls, including Mock and non-surface displayed scEDIII-Co1. Although, M cell targeting ligand Co1A conjugated only to the $\mathrm{C}$-terminus of EDIII was previously reported to enhance EDIII-specific immune responses in systemic and mucosal compartments in E.coli expression system [24], it seemed that such effect was not relevant in yeast expression system and elicitation of immune response depended mostly on the surface display strategy. This continued to increase until 32 days post last immunization. Several instances of yeast surface displayed recombinant vaccines eliciting significant humoral and cellular immune response have been reported. A study using a neutralizing epitope fragment of ApxIIA toxin (ApxIIA\#5) of the Korean A. pleuropneumoniae serotype 2 strain displayed on the yeast cell surface [34] revealed elicitation of significant humoral immune responses. H5N1 hemagglutinin surface-presented yeast triggered both humoral and cell-mediated immunity in mice [51]. Invoking a mucosal immune response through antigen-specific sIgA production is one of the prerequisites of a successful oral vaccine [52]. Fecal sIgA levels were elevated slightly in surface displayed Co1-scEDIII-AGA fed mice, around 25 days post last oral dose, reflecting stimulation by exposure of PPs to the antigen Co1-scEDIII-AGA. In the mucosal immune system, gut-associated lymphoid tissue, including PPs, play an important role in the induction of antigen-specific immune responses in the gut. Although the sIgA titers were low, upon further analysis, the numbers of AFCs in the lymphocytes isolated from the spleen and PPs differed considerably between Co1-scEDIII-AGAadministered mice and the Mock control, as well as the scEDIII-Co1 administered counterparts. Interestingly, this study showed that the IgG and IIgA titers increased significantly upon booster dose immunization of purified E. coli-expressed scEDIII antigen. This was in correlation with our earlier observation that a single intraperitoneal injection of purified scEDIII induced a rapid surge in an immune response, especially in WC-fed mice [19].

Although the immune responses in mice fed with WC surface displayed EDIII yeast were significantly higher than those fed with Mock yeast or intracellularly expressed EDIII yeast during the vaccination period, there were still high variations among mice in this group
(Fig. 5). In our previous study [19], high variations in immune responses (IgG and $\operatorname{IgA}$ ) were also observed in the mouse group fed with whole yeast cells expressing LTB-scEDIII. In spite of the advantage of protecting the antigen from gastric degradation, feeding whole cells inadvertently reduces the amount of the antigen available for uptake in Peyer's patches. Based on these consistent results, we believe that the variations are ultimately related to differential uptake of the antigen in different mice.

Limitations of most oral vaccines are associated with problems linked to antigen breakdown in the harsh gastric environment, as well as to the highly tolerogenic gut environment. The relative elicitation of both humoral and mucosal immune responses confirmed the suppression of oral tolerance. This was in correlation with earlier reports of efficient antigen delivery into PPs through oral administration of $\mathrm{M}$ cell targeting ligand Co1-conjugated EDIII antigens [24].

Due to the presence of genetically distinct complex serotypes, an ideal dengue vaccine should induce a lifelong balance and lasting immunity against all four DENV serotypes. Despite some limitations of scEDIIII as a potential dengue vaccine candidate [53], yeast-expressed scEDIII induces specific antibodies against EDIIIs from all four serotypes [54] and, together with its small size, scEDIII is an attractive tetravalent dengue vaccine model for dengue vaccine development. Our previous study involving the scEDIII gene, also applied in the current study, showed that recombinant scEDIII produced neutralizing antibodies upon oral immunization [19]. Thus, the current vaccine candidate, surface displayed Co1scEDIII-AGA, represents an efficient dengue vaccine candidate, possessing neutralization capability towards all four serotypes.

With the most affected regions being resource limited countries, an effective vaccine must be economical to meet the demands of mass immunization. Thus, oral vaccines are particularly attractive $[52,55]$. The current study presents a cost-effective, efficacious oral vaccination strategy for the administration of S. cerevisiae-surface displayed Co1-scEDIII-AGA. Without using complicated processing techniques, the described construct not only elicits both systemic and mucosal immune responses, but the antibodies produced due to its oral immunization are believed to possess neutralizing ability against all dengue virus serotypes.

\section{Conclusions}

This study represents an enhancement to our previous attempt to design economical and effective dengue vaccine candidates. Oral administration of whole yeast cells engineered to surface display the targeting 
ligand fused synthetic antigen Co1-scEDIII-AGA produced a stronger immune response compared to nondisplayed Co1-scEDIII. In addition, Co1-scEDIII-AGA fed mice further elicited a significant immune response, both humoral as well as mucosal, upon intraperitoneal booster dose. These findings suggest that the yeast surface displayed preparations of Co1-scEDIII-AGA recombinant protein administered in the form of whole yeast cells show promise as a potent oral vaccine candidate against dengue virus infection.

\section{Authors' contributions}

$\mathrm{DK}, J \mathrm{~B}, \mathrm{HJ}, \mathrm{NL}, \mathrm{JP}$, and $\mathrm{YJ}$ contributed to the conception or design of the work. $\mathrm{HJ}$ and NL performed cloning, expression, and antigen preparation. JB and $\mathrm{HJ}$ performed immunization work and ELISAs. JB, HJ, and JP performed the ELIS$\mathrm{POT}$ assay. JB contributed to data acquisition and analysis and wrote the original manuscript. All authors provided inputs for the initial draft and approved the final version. DK and YJ contributed towards critical reviewing and editing of the manuscript. All authors read and approved the final manuscript.

\section{Author details}

${ }^{1}$ Institute for Molecular Biology and Genetics, Department of Molecular Biology, Department of Bioactive Material Sciences, Chonbuk National University, Jeonju, Jeollabuk-do 54896, Republic of Korea. ${ }^{2}$ Department of Biology, College of Sciences, Hue University, Hue, Vietnam.

\section{Acknowledgements}

We are thankful to the Institute of Molecular Biology and Genetics at Chonbuk National University for kindly providing the facilities for this research. We also acknowledge Center for University-Wide Research Facilities at Chonbuk National University for providing confocal microscopy facilities.

\section{Competing interests}

The authors declare that the research was conducted in the absence of any commercial or financial relationships that could be construed as a potential competing interests.

\section{Availability of data and materials}

All data generated or analyzed during this study are included in this published article.

\section{Consent for publication}

Not relevant.

\section{Ethics approval and consent to participate}

Experimental procedures involving laboratory animals strictly adhered to the guidelines set out by the Institutional Animal Care and Use Committee of the Chonbuk National University (Approval Number: CBU 2015-0004).

\section{Funding}

This work was supported in part by the Basic Science Research Program through the National Research Foundation of Korea (NRF) funded by the Ministry of Education (2017R1A6A1A03015876) and the NRF Grant by MSIP (2015R1A2A1A10055684).

\section{Publisher's Note}

Springer Nature remains neutral with regard to jurisdictional claims in published maps and institutional affiliations.

Received: 2 May 2018 Accepted: 10 September 2018

Published online: 14 September 2018

\section{References}

1. Achee NL, Gould F, Perkins TA, Reiner RC Jr, Morrison AC, Ritchie SA, Gubler DJ, Teyssou R, Scott TW. A critical assessment of vector control for dengue prevention. PLoS Negl Trop Dis. 2015;9:e0003655.

2. WHO. Dengue: guidelines for diagnosis, treatment, prevention, and control. Spec Program Res Train Trop Dis. 2009;409:147. WHO reference number:WHO/HTM/NTD/DEN/2009.1.

3. Bhatt S, Gething PW, Brady OJ, Messina JP, Farlow AW, Moyes CL, Drake JM, Brownstein JS, Hoen AG, Sankoh O, et al. The global distribution and burden of dengue. Nature. 2013;496:504-7.

4. McArthur MA, Sztein MB, Edelman R. Dengue vaccines: recent developments, ongoing challenges and current candidates. Expert Rev Vaccines. 2013;12:933-53.

5. Yauch LE, Shresta S. Dengue virus vaccine development. Adv Virus Res. 2014;88:315-72.

6. Hadinegoro SR, Arredondo-Garcia JL, Capeding MR, Deseda C, Chotpitayasunondh T, Dietze R, Muhammad Ismail HI, Reynales H, Limkittikul K, Rivera-Medina DM, et al. Efficacy and long-term safety of a dengue vaccine in regions of endemic disease. N Engl J Med. 2015;373:1195-206.

7. Malisheni M, Khaiboullina SF, Rizvanov AA, Takah N, Murewanhema G, Bates M. clinical efficacy, safety, and immunogenicity of a live attenuated tetravalent dengue vaccine (CYD-TDV) in children: a systematic review with meta-analysis. Front Immunol. 2017;8:863.

8. Katzelnick LC, Fonville JM, Gromowski GD, Bustos Arriaga J, Green A, James SL, Lau L, Montoya M, Wang C, VanBlargan LA, et al. Dengue viruses cluster antigenically but not as discrete serotypes. Science. 2015:349:1338-43.

9. Halstead SB. Neutralization and antibody-dependent enhancement of dengue viruses. Adv Virus Res. 2003;60:421-67.

10. Lindenbach BD, Rice CM. Molecular biology of flaviviruses. Adv Virus Res. 2003;59:23-61.

11. Chen Y, Maguire T, Hileman RE, Fromm JR, Esko JD, Linhardt RJ, Marks RM. Dengue virus infectivity depends on envelope protein binding to target cell heparan sulfate. Nat Med. 1997;3:866-71.

12. Chen $Y C$, Wang $S Y$, King CC. Bacterial lipopolysaccharide inhibits dengue virus infection of primary human monocytes/macrophages by blockade of virus entry via a CD14-dependent mechanism. J Virol. 1999;73:2650-7.

13. Modis Y, Ogata S, Clements D, Harrison SC. Structure of the dengue virus envelope protein after membrane fusion. Nature. 2004;427:313-9.

14. Modis Y, Ogata S, Clements D, Harrison SC. Variable surface epitopes in the crystal structure of dengue virus type 3 envelope glycoprotein. J Virol. 2005;79:1223-31.

15. Guzman MG, Hermida L, Bernardo L, Ramirez R, Guillen G. Domain III of the envelope protein as a dengue vaccine target. Expert Rev Vaccines. 2010;9:137-47.

16. Whitehead SS, Blaney JE, Durbin AP, Murphy BR. Prospects for a dengue virus vaccine. Nat Rev Microbiol. 2007;5:518-28.

17. Leng CH, Liu SJ, Tsai JP, Li YS, Chen MY, Liu HH, Lien SP, Yueh A, Hsiao $\mathrm{KN}$, Lai LW, et al. A novel dengue vaccine candidate that induces cross-neutralizing antibodies and memory immunity. Microbes Infect. 2009;11:288-95.

18. Nguyen NL. Development of recombinant dengue vaccines using Saccharomyces cerevisiae and cloning and characterization of fungal polyketide synthase genes in the pathogenic fungus Cladosporium phlei. Jeonju: Chonbuk National University, Department of Molecular Biology; 2013.

19. Bal J, Luong NN, Park J, Song KD, Jang YS, Kim DH. Comparative immunogenicity of preparations of yeast-derived dengue oral vaccine candidate. Microb Cell Fact. 2018;17:24.

20. Bucarey SA, Noriega J, Reyes P, Tapia C, Saenz L, Zuniga A, Tobar JA. The optimized capsid gene of porcine circovirus type 2 expressed in yeast forms virus-like particles and elicits antibody responses in mice fed with recombinant yeast extracts. Vaccine. 2009;27:5781-90.

21. Wang $M$, Gao Z, Zhang Z, Pan L, Zhang Y. Roles of $M$ cells in infection and mucosal vaccines. Hum Vaccin Immunother. 2014;10:3544-51.

22. Kim SH, Jang YS. Antigen targeting to M cells for enhancing the efficacy of mucosal vaccines. Exp Mol Med. 2014;46:e85.

23. Nguyen NL, So KK, Kim JM, Kim SH, Jang YS, Yang MS, Kim DH. Expression and characterization of an $\mathrm{M}$ cell-specific ligand-fused dengue virus tetravalent epitope using Saccharomyces cerevisiae. J Biosci Bioeng. 2015;119:19-27. 
24. Kim SH, Jung DI, Yang IY, Jang SH, Kim J, Truong TT, Pham TV, Truong NU, Lee KY, Jang YS. Application of an M-cell-targeting ligand for oral vaccination induces efficient systemic and mucosal immune responses against a viral antigen. Int Immunol. 2013;25:623-32.

25. Bernstein MB, Chakraborty M, Wansley EK, Guo Z, Franzusoff A, Mostbock S, Sabzevari H, Schlom J, Hodge JW. Recombinant Saccharomyces cerevisiae (yeast-CEA) as a potent activator of murine dendritic cells. Vaccine. 2008;26:509-21.

26. Wadle A, Held G, Neumann F, Kleber S, Wuellner B, Asemissen AM, Kubuschok B, Scheibenbogen C, Breinig T, Meyerhans A, Renner C. Cross-presentation of HLA class I epitopes from influenza matrix protein produced in Saccharomyces cerevisiae. Vaccine. 2006;24:6272-81.

27. Howland SW, Tsuji T, Gnjatic S, Ritter G, Old LJ, Wittrup KD. Inducing efficient cross-priming using antigen-coated yeast particles. J Immunother 2008;31:607-19.

28. Liu Z, Zhou G, Ren C, Xu K, Yan Q, Li X, Zhang T, Zhang Z. Oral administration of myostatin-specific recombinant Saccharomyces cerevisiae vaccine in rabbit. Vaccine. 2016;34:2378-82.

29. Cherf GM, Cochran JR. Applications of yeast surface display for protein engineering. Methods Mol Biol. 2015;1319:155-75.

30. Boder ET, Wittrup KD. Yeast surface display for directed evolution of protein expression, affinity, and stability. Methods Enzymol. 2000;328:430-44

31. Wadle A, Held G, Neumann F, Kleber S, Wuellner B, Asemissen AM, Kubuschok B, Scheibenbogen C, Breinig T, Meyerhans A, Renner C. Cross-presentation of HLA class I epitopes from influenza matrix protein produced in Saccharomyces cerevisiae. Vaccine. 2006;24:6272-81.

32. Zhu K, Chi Z, Li J, Zhang F, Li M, Yasoda HN, Wu L. The surface display of haemolysin from Vibrio harveyi on yeast cells and their potential applications as live vaccine in marine fish. Vaccine. 2006;24:6046-52.

33. Wasilenko Jamie L, Sarmento L, Spatz S, Pantin-Jackwood M. Cell surface display of highly pathogenic avian influenza virus hemagglutinin on the surface of Pichia pastoris cells using a-agglutinin for production of oral vaccines. Biotechnol Prog. 2009;26:542-7.

34. Kim JM, Jung DI, Eom YJ, Park SM, Yoo HS, Jang YS, Yang MS, Kim DH. Surface-displayed expression of a neutralizing epitope of ApxllA exotoxin in Saccharomyces cerevisiae and oral administration of it for protective immune responses against challenge by Actinobacillus pleuropneumoniae. Biosci Biotechnol Biochem. 2010;74:1362-7.

35. Shibasaki S, Aoki W, Nomura T, Miyoshi A, Tafuku S, Sewaki T, Ueda M. An oral vaccine against candidiasis generated by a yeast molecular display system. Pathog Dis. 2013;69:262-8.

36. Park S-M, Mo A-Y, Lim J-G, Chung H-J, Kim T-G, Kim K-J, Cho D-H, Yang M-S, Kim D-H. Surface displayed expression of a neutralizing epitope of spike protein from a Korean strain of porcine epidemic diarrhea virus. Biotechnol Bioprocess Eng. 2007;12:690-5.

37. Shin YM, Kwon TH, Kim KS, Chae KS, Kim DH, Kim JH, Yang MS. Enhanced iron uptake of Saccharomyces cerevisiae by heterologous expression of a tadpole ferritin gene. Appl Environ Microb. 2001;67:1280-3.

38. Gietz D, St Jean A, Woods RA, Schiestl RH. Improved method for high efficiency transformation of intact yeast cells. Nucleic Acids Res. 1992;20:1425

39. Bender A, Pringle JR. Multicopy suppression of the cdc24 budding defect in yeast by CDC42 and three newly identified genes including the rasrelated gene RSR1. Proc Natl Acad Sci U S A. 1989;86:9976-80.
40. Kim SH, Seo KW, Kim J, Lee KY, Jang YS. The M cell-targeting ligand promotes antigen delivery and induces antigen-specific immune responses in mucosal vaccination. J Immunol. 2010;185:5787-95.

41. Kim SH, Jung DI, Yang IY, Kim J, Lee KY, Nochi T, Kiyono H, Jang YS. M cells expressing the complement C5a receptor are efficient targets for mucosal vaccine delivery. Eur J Immunol. 2011;41:3219-29.

42. De Smet R, Allais L, Cuvelier CA. Recent advances in oral vaccine development: yeast-derived beta-glucan particles. Hum Vaccin Immunother. 2014:10:1309-18.

43. Schreuder MP, Deen C, Boersma WJ, Pouwels PH, Klis FM. Yeast expressing hepatitis $B$ virus surface antigen determinants on its surface: implications for a possible oral vaccine. Vaccine. 1996;14:383-8.

44. Ramasamy V, Arora U, Shukla R, Poddar A, Shanmugam RK, White LJ, Mattocks MM, Raut R, Perween A, Tyagi P, et al. A tetravalent virus-like particle vaccine designed to display domain III of dengue envelope proteins induces multi-serotype neutralizing antibodies in mice and macaques which confer protection against antibody dependent enhancement in AG129 mice. PLoS Negl Trop Dis. 2018;12:e0006191.

45. Kim MY, Van Dolleweerd C, Copland A, Paul MJ, Hofmann S, Webster GR, Julik E, Ceballos-Olvera I, Reyes-Del Valle J, Yang MS, et al. Molecular engineering and plant expression of an immunoglobulin heavy chain scaffold for delivery of a dengue vaccine candidate. Plant Biotechnol J. 2017;15:1590-601.

46. Cerutti A. Location, location, location: B-cell differentiation in the gut lamina propria. Mucosal Immunol. 2008;1:8-10.

47. Kuolee R, Chen W. M cell-targeted delivery of vaccines and therapeutics. Expert Opin Drug Deliv. 2008:5:693-702.

48. Schreuder MP, Brekelmans S, van den Ende H, Klis FM. Targeting of a heterologous protein to the cell wall of Saccharomyces cerevisiae. Yeast. 1993;9:399-409.

49. Tanaka T, Yamada R, Ogino C, Kondo A. Recent developments in yeast cell surface display toward extended applications in biotechnology. Appl Microbiol Biotechnol. 2012;95:577-91.

50. Lei H, Jin S, Karlsson E, Schultz-Cherry S, Ye K. Yeast surface-displayed H5N1 avian influenza vaccines. J Immunol Res. 2016;2016:4131324.

51. Pavot V, Rochereau N, Genin C, Verrier B, Paul S. New insights in mucosal vaccine development. Vaccine. 2012;30:142-54.

52. Wang L, Coppel RL. Oral vaccine delivery: can it protect against nonmucosal pathogens? Expert Rev Vaccines. 2008;7:729-38.

53. Chen HW, Liu SJ, LiYS, Liu HH, Tsai JP, Chiang CY, Chen MY, Hwang CS, Huang CC, Hu HM, et al. A consensus envelope protein domain III can induce neutralizing antibody responses against serotype 2 of dengue virus in non-human primates. Arch Virol. 2013;158:1523-31.

54. Nguyen NL, Kim JM, Park JA, Park SM, Jang YS, Yang MS, Kim DH. Expression and purification of an immunogenic dengue virus epitope using a synthetic consensus sequence of envelope domain III and Saccharomyces cerevisiae. Protein Expr Purif. 2013;88:235-42.

55. Levine MM. Immunogenicity and efficacy of oral vaccines in developing countries: lessons from a live cholera vaccine. BMC Biol. 2010;8:129.

\footnotetext{
Ready to submit your research? Choose BMC and benefit from:

- fast, convenient online submission

- thorough peer review by experienced researchers in your field

- rapid publication on acceptance

- support for research data, including large and complex data types

- gold Open Access which fosters wider collaboration and increased citations

- maximum visibility for your research: over $100 \mathrm{M}$ website views per year
}

At BMC, research is always in progress.

Learn more biomedcentral.com/submissions 